\title{
Valproic Acid: A Summary of Indian Epilepsy Society-Consensus Document
}

\author{
Man Mohan Mehndiratta ${ }^{a}$, Sanjeev V. Thomas ${ }^{b}$, Neeraj N. Baheti ${ }^{c}$, Atma Ram Bansal $^{\mathrm{d}}$, Sanjeev Bhoi ${ }^{\mathrm{e}}$, \\ Deepanshu Dubey ${ }^{\mathrm{e}}$, Sheffali Gulati ${ }^{\mathrm{f}}$, Satish Jain ${ }^{\mathrm{g}}$, Sita Jayalakshmi ${ }^{\mathrm{h}}$, Jayantee Kalita ${ }^{\mathrm{e}}$, \\ Parampreet S. Kharbanda ${ }^{\mathrm{i}}$, Chanda Kulkarni ${ }^{\mathrm{j}}$, Bindu Menon ${ }^{\mathrm{k}}$, Usha Kant Misra ${ }^{\mathrm{e}}$, P. Satishchandra ${ }^{1}$, \\ Vinod S. Saxena $^{\mathrm{m}}$, Suvasini Sharma ${ }^{\mathrm{n}}$, Gagandeep Singh ${ }^{\circ}$, Sanjib Sinha ${ }^{\mathrm{p}}$, Manjari Tripathi ${ }^{\mathrm{f}}$, \\ Sudhindra Vooturi ${ }^{\mathrm{q}}$, Nandan Yardi ${ }^{\mathrm{r}}$ \\ a Janakpuri Super Speciality Hospital, New Delhi \\ ${ }^{\mathrm{b}}$ Sree Chitra Tirunal Institute for Medical Sciences and Technology, Thiruvananthapuram \\ ${ }^{\mathrm{c}}$ Central India Institute of Medical Sciences, Nagpur 440010, Maharashtra \\ ${ }^{\mathrm{d}}$ Medanta-The Medicity Gurgaon, Haryana \\ e Sanjay Gandhi Post Graduate Institute of Medical Sciences, Raebareli Road, Lucknow \\ ${ }^{\mathrm{f}}$ All India Institute of Medical Sciences, New Delhi \\ ${ }^{g}$ Director, Indian Epilepsy Centre, New Delhi \\ ${ }^{\mathrm{h}}$ Krishna Institute of Medical Sciences, Secunderabad, Telangana \\ ${ }^{i}$ Postgraduate Institute of Medical Education E' Research (PGIMER), Chandigarh \\ ${ }^{\mathrm{j}}$ SAKRA World Hospital, Bangalore, India \\ ${ }^{\mathrm{k}}$ Apollo Speciality Hospitals, Nellore \\ ${ }^{1}$ National Institute of Mental Health \& Neuro Sciences (NIMHANS), Bengaluru, Karnataka \\ ${ }^{\mathrm{m}}$ Life Trustee Indian Epilepsy Association - 18th International Epilepsy Congress Trust, Delhi \\ ${ }^{\mathrm{n}}$ Lady Hardinge Medical College and Associated Kalawati Saran Children's Hospital, New Delhi \\ ${ }^{\circ}$ Dayanand Medical College, Ludhiana \\ ${ }^{\mathrm{p}}$ NIMHANS, Banglore \\ ${ }^{\mathrm{q}}$ Krishna Institute of Medical Sciences, Minister Road, Secunderabad - 03, Telangana, India \\ ${ }^{\mathrm{r}} \mathrm{BJ}$ Medical College (MUHS), Pune
}

\section{Introduction}

Valproic acid (VPA) is a well established anticonvulsant drug for the last 40 years and is now a part of more than 130 national health care programmes. Since 1988 , it has regularly featured as an anticonvulsant in the WHO Model List of Essential Medicines [1]. The current NICE Guidelines recommend Valproic Acid as the first-line of treatment for Idiopathic Generalized Epilepsies (IGEs) in children, young people and adults but should be used with caution in pregnant women. [2]

\section{Mechanism of Action}

- VPA stimulates glutamic acid dehydrogenase, the enzyme primarily involve in GABA synthesis, and increases GABA concentrations within the brain. Thus, reducing excessive neuronal firing. [3], [4]

- VPA attenuates NMDA receptor mediated excitation and inhibits sodium and calcium channel function which reduces glutamatergic transmission with increased brain levels of GABA

- VPA produces weak inhibition of voltage-gated sodium channels, leading to prolongation of refractory period of high frequency neuronal firing thus limiting the frequency of neuronal depolarization. [5] The blockade of T-type calcium channels is also said to contribute to its anticonvulsant effects.
- VPA at lower concentrations is shown to diminish high frequency repetitive firing of action potentials of central neurons critically involved in its activity in generalized tonicclonic seizures. [6]

- Putative mechanisms involved in early and late anticonvulsant effects [7] come from the quick accesses to extracellular sites e.g. ion channels and slow access to intracellular sites e.g. GABA synthesis following acute administration of VPA.

\section{Pharmacokinetics}

- VPA is almost completely absorbed with enteric-coated formulations are slowly absorbed - reaching peak levels 3 to 5 hours after intake.

- The therapeutic blood concentrations of VPA are $50-100 \mathrm{~g} / \mathrm{ml}$ with a half-life of 6-15 hours.

- Being highly lipophilic, it concentrates in the liver and cross the blood brain barrier to give high concentrations in the brain.

- VPA is extensively metabolized through oxidative and conjugation mechanisms. The metabolites are biologically inactive.

- Its half-life in various age groups is different - neonates (in first week of life): 40-45 hours; neonates <10 days: 10-67 hours; infants and children > 2months: 7-13 hours; children and adolescents 2-14 years: 9 hours; and adults: 9-16 hours. 
Table 1

Guidelines for use of Valproic acid in epilepsy.

\begin{tabular}{|c|c|c|}
\hline Type of seizure & ILAE (013) recommendations & NICE recommendations \\
\hline GTCS & VPA is possibly efficacious as monotherapy & VPA as first line treatment \\
\hline Absence seizures & $\begin{array}{l}\text { VPA and ETM are efficacious as monotherapy } \\
\text { as initial monotherapy for children with newly } \\
\text { diagnosed or untreated absence seizures }\end{array}$ & VPA as the first line treatment \\
\hline $\begin{array}{l}\text { Benign childhood epilepsy } \\
\text { with centretemporal spikes }\end{array}$ & VPA and CBZ are efficacious as monotherapy. & $\begin{array}{l}\text { VPA as the first line treatment along } \\
\text { with CBZ, LTG, OXC, LEV }\end{array}$ \\
\hline Juvenile Mycoclonic Epilepsy & $\begin{array}{l}\text { VPA and TPM are potentially efficacious/effective } \\
\text { for patients with newly diagnosed JME }\end{array}$ & $\begin{array}{l}\text { VPA as the first line treatment along } \\
\text { with LTG, LEV, TPM }\end{array}$ \\
\hline Partial Seizures & $\begin{array}{l}\text { VPA is possibly efficacious/effective as initial } \\
\text { monotherapy for children with newly diagnosed } \\
\text { or untreated partial onset seizures. }\end{array}$ & $\begin{array}{l}\text { - Offer CBZ or LTG as first line treatment } \\
\text { - VPA can be offered as adjunctive } \\
\text { treatment }\end{array}$ \\
\hline Myoclonic & Not mentioned separately & VPA as the first line drug \\
\hline Atonic seizures & Not mentioned separately & VPA as the first line drug \\
\hline \multicolumn{3}{|c|}{$\begin{array}{l}\text { Other seizures/epileptic encephalopathies } \\
\text { where VPA has been found to be effective: } \\
\text { 1. West Syndrome } \\
\text { 2. Dravet Syndrome } \\
\text { 3. Benign myoclonic epilepsy of infancy } \\
\text { 4. Lennox-Gastaut syndrome } \\
\text { 5. Myoclonic astatic epilepsy } \\
\text { 6. Continuous spike and wave during sleep } \\
\text { 7. Landau Klefner syndrome }\end{array}$} \\
\hline
\end{tabular}

Table 2

Interaction of VPA with other commonly used drugs.

\begin{tabular}{|c|c|}
\hline Drugs that may decrease Valproic acid levels & Carbapenems, Ethosuximide, Mefloquine, Protease inhibitors, Rifampicin \\
\hline Drugs that may increase Valproic acid levels & Chlorpromazine, Felbamate, Salicylates, Topiramate \\
\hline Drugs whose levels may increase with Valproic acid intake & Barbiturates, Carbamazepine, Lamotrigine, Lorazepam, Risperidone, Rufinamide, Zidovudine \\
\hline Drugs whose levels may decrease with Valproic acid intake & Phenytoin/Fosphenytoin, Olanazipine Oxcarbazepine \\
\hline
\end{tabular}

\section{Valproic Acid in Idiopathic Generalized Epilepsy (IGE)}

- As per AAN Guidelines, VPA is effective in the treatment of Absence seizures, primary generalized-onset tonic-clonic seizures especially Juvenile Myoclonic Epilepsy.

- VPA considered as the drug of choice in patients with IGEs, especially when given as the sole anti-epileptic drug. [8,9]

- While the other AEDs like carbamazepine, Oxcarbazepine, Lamotrigine and phenytoin may aggravate seizures in certain cases, VPA has very low risk of seizure aggravation.

- VPA is found to be more effective than Lamotrigine and better tolerated than Topiramate in generalized or unclassified epilepsy. [10]

- It is believed that if the daily dose of VPA does not exceed $40 \mathrm{mg} / \mathrm{kg}$ or $2.5 \mathrm{~g}$, then it is singularly free from serious side effects. [11]

- It can also be recommended as first-line monotherapy in IGEs with multiple seizure types. [12]

- It can also be prescribed as an initial conservative treatment option in newly diagnosed patients in whom the nature of epilepsy syndrome is still undetermined. [13]

- However, it should be avoided in women of childbearing age due to its safety profile with concerns of teratogenesis and weight gain.

\section{Valproic Acid in Absence Seizures}

- VPA monotherapy was found to be effective in controlling absence seizures and should be given adequate trial before adding or switching to another AED. [14]
Combination therapy with ESM and VPA should be considered for patients whose absence seizures do not respond to standard therapeutic measures. [15]

- Its combination with a low dose Lamotrigine appears to have synergistic effect in typical absence seizures. [16]

- The patients with primary generalized epilepsy responds better than those with diffuse cerebral damage or generalized epilepsy with focalization. Therefore, VPA is the drug of choice for the prevention of ASE recurrence. [17]

\section{Valproic Acid in Generalized Tonic-Clonic Seizures (GTCS)}

- A study shows non-statistical trend towards a better response with VPA compared with CBZ. [18]

- VPA monotherapy is very effective for both seizure outcome control and photosensitivity reduction in adolescents with epilepsy with generalized tonic-clonic seizures only. [19]

\section{Valproic acid in Juvenile Myoclonic Epilepsy (JME)}

- VPA is among the most efficacious drug in JME but may be poorly tolerated by some.

Table 3

Effects of Valproic Acid on other antiepileptic drugs.

\begin{tabular}{ll}
\hline Anti-epileptic drug & Effect on its level on co-administration with VPA \\
\hline Lamotrigine & Lamotrigine levels increase by $85 \%$ \\
Topiramate & Topiramate levels stay unchanged \\
Felbamate & Felbamate levels increase by $27 \%$ \\
\hline
\end{tabular}


Table 4

Adverse effects of valproic acid that can be of major concern to women with epilepsy.

\begin{tabular}{l} 
Hair Loss \\
Tremor \\
Obesity \\
Menstrual irregularity \\
Polycystic ovarian syndrome \\
Infertility \\
Osteoporosis \\
Teratogenic effects and impaired cognitive development in \\
$\quad$ prenatally exposed offspring \\
\hline
\end{tabular}

- Acute treatment for most myoclonic seizures starts with a BZD; however, as a second step, intravenous VPA can be administered at a high loading dose to rapidly achieve therapeutic levels.

\section{Valproic Acid in Focal Epilepsy}

- VPA should not be the drug of choice for focal seizures at all with better treatment options available.

\section{Valproic Acid in Status Epilepticus}

- Intravenous VPA is an emerging alternative in SE patients who are resistant to the first-line drugs such as BZD.

- The recommended dose of VPA is $15-45 \mathrm{mg} / \mathrm{kg}$ bolus followed by $1-3 \mathrm{mg} / \mathrm{kg}$ in infusion.

- Safety data is also encouraging with the incidence of adverse reaction below $1 \%$.

\section{Valproic Acid in Childhood Epilepsies}

- VPA is a drug of choice for children with newly diagnosed epilepsy, idiopathic generalized epilepsy, absence seizures along with myoclonic seizures, juvenile myoclonic seizures and atonic seizures or with multiple seizure types and photosensitive epilepsies

- Intravenous VPA may be effective for the treatment of convulsive and non-convulsive status epilepticus that is refractory to conventional drugs. [20]

- VPA has been found to be effective in febrile seizure prophylaxis but is not recommended due to the self-limiting and benign nature of the disease.

- In limited literature, VPA has been found to be effective for treating infantile spasms.

- It has also been reported to be effective in children with LennoxGastuat Syndrome (LGS) and suggested as the first-line treatment for children with LGS myoclonic astatic epilepsy.

- VPA has been proposed as an adjunctive AED among patients with continuous spike and wave during sleep and Landau Klefner Syndrome.

- There is only limited data to show the efficacy of Valproic Acid in children with Status Epilepticus.

- In infants, the potential benefits of VPA should be carefully weighed against the liver toxicity and gastrointestinal intolerance.

\section{Adverse Effects of Valproic Acid}

- The most common adverse effects include sleep disturbances, dizziness, abdominal pain/dyspepsia, rash, increased appetite/ weight gain, tremors, alopecia, and hirsutism.

- The more serious adverse effects include hepatotoxicity (idiosyncratic Reye's like syndrome), hyperammonemic encephalopathy, mitochondrial toxicity, pancreatitis, Steven's Johnson Syndrome/toxic epidermal necrolysis and aplastic anemia.

\section{VPA-induced Hepatotoxicity}

- It is rare but a serious complication.

- It is an idiosyncratic reaction that occurs in genetically predisposed individuals - those less than 2 years of age and harbouring the polymerase gamma 1 (POLG1) mutation, a feature of a mitochondrial disorder called Alpers-Huttenlocher syndrome.

- Three clinical forms of Hepatotoxicity can occur with VPA: Hyperammonemia manifests as progressive and episodic confusion followed by obtundation and coma, with minimal or no evidence of hepatic injury; Acute hepatocellular injury with jaundice typically accompanied by hepatocellular or mixed pattern of enzyme elevations; and Reye-like syndrome described in children on VPA that develop fever and lethargy followed by confusion, stupor and coma.

- VPA-induced hepatitis in children less than 2 years of age is invariably fatal. Therefore, it is important to screen the high-risk individuals for POLG1 mutation before commencing VPA treatment.

- Risk factors for developing hepatitis are - age below 2 years, use of VPA as polytherapy, children with mental retardation, evidence of any pre-existing liver disease or elevated liver enzyme, co-existence of certain metabolic defects e.g. oxidation disorders and mitochondrial diseases.

- VPA-induced hepatotoxicity can be avoided if the drug is used as monotherapy whenever possible especially in children under 3 years, keep minimal possible dose required for seizure control, avoid salicylates, and avoid VPA in patients with liver disease or metabolic disorders involving the urea cycle, organic acidaemias, mitochondrial disorders, free radical scavenger deficiencies, carnitine or medium chain acyl coenzyme A deficiency.

- In the event of features of an adverse effect, the following tests should be performed: complete blood count, liver function tests, serum electrolytes, plasma ammonia and serum Valproic acid levels.

\section{Contraindications of Valproic Acid}

- Known mitochondrial disorders caused by mutations in mitochondrial DNA polymerase gamma (POLG) e.g. Alpers-Huttenlocher syndrome (AHS) or children less than 2 years of age suspected of having a POLG-related disorder.

- Urea Cycle Defect

- Hypersensitivity to Valproic acid, divalproex, derivatives or any component of the formulation.

- Hepatic disease or significant hepatic impairment

\section{Special Considerations}

- Children less than 2 years of age: Neonates, infants and children less than 2 years of age are at considerable increased risk for Hepatotoxicity, especially those on anticonvulsant polytherapy, with congenital metabolic disorders, with severe seizures disorders and mental retardation, or with organic brain disease.

- Adolescent girls: The adverse effects including hirsutism, weight gain and polycystic ovarian syndrome assumes greater significance among adolescent girls. These should be balanced against the therapeutic benefits and discussed with the family before initiating VPA among adolescent girls.

- Pregnancy and lactation. VPA intake during pregnancy is contraindicated. It has been associated with increased risk of neural tube defects, microcephaly, heart defects, and diaphragmatic hernia. Also, it has been shown that low doses of VPA are 
secreted in breast milk. However, these VPA levels in breast milk are insignificant and do not impact infant's growth or development. Nonetheless, it is recommended that infant's breastfeeding on mothers taking VPA should be monitored for jaundice and petechial rash.

\section{Management of Epilepsy in Women who are planning their pregnancies}

- Valproic acid should preferably be avoided as the first line drug for treatment of epilepsy in girls and women of reproductive age.

- Valproic acid may be avoided in obese women with history of menstrual irregularities or polycystic ovarian syndrome.

- Whenever possible Valproic acid dosage need to be maintained below $600 \mathrm{mg}$ per day for adult women.

- Valproic acid can be continued (preferably in low dose) in women in reproductive age group who are already in long term remission of epilepsy while on Valproic acid.

- Low dose Valproic acid (<600 mg per day) may be considered under certain circumstances as one of the first line drugs for treating newly diagnosed generalized epilepsy in girls and women of reproductive age group after a full discussion on its risk to the patient.

- Valproic acid can be used as a first line drug to treat age specific epilepsies of childhood such as absence or BECT that are known to resolve before puberty.

- Valproic acid can be used in girls and women who are unlikely to become pregnant (girls with epileptic encephalopathies or significant comorbidities and women who had undergone sterilization or have become menopausal).

- All women and caregivers for girls should receive full information of the benefits and risks prior to treatment with Valproic acid.

- All women with childbearing potential need to be prescribed folic acid $5 \mathrm{mg}$ daily.

\section{Management of Pregnancy in Women who are on Valproic Acid}

- A dose reduction to less than $600 \mathrm{mg}$ per day can be considered in early pregnancy in those who are in remission.

- All pregnant women with epilepsy need to have a screening of serum alpha feto protein and a level III antenatal ultrasonography to carefully evaluate the fetal organs for any malformations.

\section{Breastfeeding by women who are using Valproic Acid}

- The benefits of VPA outweigh the potential risk from the exposure to Valproic acid through breast milk.

\section{Valproic Acid in Epilepsy with various Comorbidities}

- Mood disorders: Valproic acid has a mood stabilizing property and thus, is a drug of choice in persons with concomitant mood disorders. [21] During discontinuation of VPA, the patient may show features of affective disorders like mania or depression and should be taken care accordingly. It is particularly effective in bipolar affective disorder especially with rapid cycling and dysphoric mania. [22]

- Migraine: Studies show the effectiveness of VPA monotherapy in migraine prophylaxis including extended release of Valproic acid. $[23,24]$ It is also found to be effective in reducing headache frequency and is reasonably well tolerated in adult patients with episodic migraine.

\section{Role of Valproic Acid in Systemic Disorders}

- Thyroid disorders Majority of the reports mention minimal suppression of thyroid functions with Valproic acid. But the changes are transient and reversible with withdrawal of medication. [25]

- Obesity Valproic acid should be used with caution in obese with an advice regarding dietary and life style modifications with frequent weight monitoring.

- Polycystic ovarian syndrome: Polycystic ovarian syndrome features may developed more frequently during Valproic acid than Lamotrigine treatment and these metabolic changes are partially reversible on discontinuing Valproic acid. [26,27]

- Renal Failure: Being highly lipophilic, Valproic acid is little affected by renal disease and hemodialysis. Although Valproic acid is safe in mild-to-moderate renal failure, there are reports of acute tubular necrosis following Valproic acid use and Fanconi's syndrome. [28]

- Hepatic Failure: Valproic acid is a wide-spectrum isoenzyme inhibitor and increases its own serum level and that of other drugs sometimes causing toxicity thus dose reduction may be required in setting of hepatic dysfunction.

- Osteoporosis: Valproic acid has a strong association with osteoporosis. Therefore, calcium and vitamin D supplementation may be prescribed. In elderly population, a regular interval Dexa scan may help to detect bone loss at early stage.

- HIV Infection/AIDS Valproic acid has been associated with hepatic and multiorgan failure when used with antiretroviral drugs. [29] Valproic acid also increases the level of zidovudine. Hence taking all the things into consideration, Valproic acid should be used with caution in the treatment of epilepsy in patients with HIV/AIDS.

- Stroke: Among the antiepileptics, Valproic acid has less stroke risk than phenytoin. [30] If a patient is on long term anticoagulation, VPA may have significant interaction due to enzyme inhibition and hence should be avoided.

- Malignancies and patients on chemotherapy: Valproic acid may aggravate hematologic and other toxicities of chemotherapeutic agents due to enzyme inhibition hence it should be best avoided. [31]

- Hematological disorders: Thrombocytopenia, leukopenia, bone marrow suppression, lymphadenopathy and exacerbation of acute intermittent porphyria have been reported with Valproic acid. [32] In the presence of underlying primary hematological disorders it is best to avoid Valproic acid.

- Cardiovascular disorders: Although safe in patients with cardiac comorbidities, Valproic acid being highly protein bound can displace warfarin, digoxin, and amiodarone from its binding sites. It can lead to cardiac arrhythmias, increased anticoagulation. Hence close monitoring is needed in this situation.

- CNS infections: CNS infections like tubercular meningitis commonly present with seizures especially in adults. For acute management of seizure in tubercular meningitis, VPA can be used however for long term management it is not a very good choice in view of risk of drug interaction (enzyme inhibition) and hepatotoxicity. It is better to avoid VPA and if given, liver functions should be monitored at regular intervals. [33]

- Perioperative management: Valproic acid is well known to cause various hematological abnormalities most common being thrombocytopenia. There are conflicting reports about the bleeding risk during surgery for patients on high doses of VPA. $[34,35]$ It is advisable to monitor routine coagulation parameters before any planned surgery. 


\section{Pharmacoeconomics of Valproic Acid}

- VPA is a relatively economical AED for Generalized Epilepsies

- Immediate release preparation is significantly cheaper than the sustained release

- More economical AEDs are available for treatment of Focal Epilepsies

- For best choice of AED-specific setting, efficacy and tolerability to be viewed in concurrence to the cost.

\section{Acknowledgement to following for peer review and valuable suggestion for this document}

- Prof. Emilio Perucca-Italy

- Prof. Lim Shih Hui -Singapore

- Prof. K.P. Vinayan -India

- Prof. Sangeeta Ravat-India

\section{References}

[1] WHO List of Essential Medicines. CH1211 Geneva, Switzerland. 2015; 5.

[2] Nunes VD, Sawyer L, Neilson J, Sarri G, Cross JH. Diagnosis and management of the epilepsies in adults and children: Summary of updated NICE guidance. BMJ. 2012;344:e281

[3] Ahamed wb, Carmody h. Safe use of sodium valproate. Aust Prescr. 2014;37:124127.

[4] McNamara JC. Chapter 19. Pharmacotherapy of epilepsies. In: Brunton LL, Lazo JS, Parker KL, eds. In: Goodman E' Gilman's The Pharmacological Basis of Therapeutics. [11th] Edition New York: Th e Mc Grwa-Hill Companies Inc; 2005.

[5] Large $\mathrm{CH}$, Kalinichev M, Lucas A, et al. The relationship between sodium channel inhibition and anticonvulsant activity in a model of generalised seizure in the rat. Epilepsy Research. 2009;85:96-106.

[6] Wolfgang Löscher. Pharmacological effects and mechanisms of action. In-Mile stones in drug therapy Valproate, Ed: W Loscher. Indian Reprint 2007: 7-45.

[7] Wolfgang Löscher. Pharmacological effects and mechanisms of action. In-Mile stones in drug therapy Valproate, Ed: W Loscher. Indian Reprint 2007: 7-45.

[8] Sullivan JE, Dlugos DJ. Idiopathic Generalized Epilepsy. Current treatment options in neurology. 2004 May;6(3):231-242. PubMed PMID: 15043806.

[9] Patsalos PN. Properties of antiepileptic drugs in the treatment of idiopathic generalized epilepsies. Epilepsia. 2005;46(Suppl 9):140-148. PubMed PMID: 16302888.

[10] Marson AG, Al-Kharusi AM, Alwaidh M, Appleton R, Baker GA, Chadwick DW, et al. The SANAD study of effectiveness of valproate, lamotrigine, or topiramate for generalised and unclassifi able epilepsy: An unblinded randomised controlled trial. Lancet. 2007 Mar 24;369(9566):1016-1026. PubMed PMID; 17382828. Pubmed Central PMCID: 2039891.

[11] Covanis A, Gupta AK, Jeavons PM. Sodium valproate: Monotherapy and polytherapy. Epilepsia. 1982 Dec;23(6):693-720. PubMed PMID: 6816580.

[12] Karceski S, Morrell MJ, Carpenter D. Treatment of epilepsy in adults: Expert opinion, 2005. Epilepsy E behavior: EG'B. 2005 Sep;7(Suppl 1):S1-S64. quiz S5-7. PubMed PMID: 16102515.

[13] Trinka E, Marson AG, Van Paesschen W, Kalviainen R, Marovac J, Duncan B, et al. KOMET: An unblinded, randomised, two parallel-group, stratified trial comparing the effectiveness of levetiracetam with controlled-release carbamazepine and extended release sodium valproate as monotherapy in patients with newly diagnosed epilepsy. Journal of neurology neurosurgery and psychiatry. 2013 Oct;84(10):1138-1147. PubMed PMID: 22933814.

[14] Bourgeois B, Beaumanoir A, Blajev B, de la Cruz N, Despland PA, Egli M, et al. Monotherapy with valproate in primary generalized epilepsies. Epilepsia. 1987;28(Suppl 2):S8-S11. PubMed PMID: 3121293.

[15] Rowan AJ, Meijer JW, de Beer-Pawlikowski N, van der Geest P, Meinardi H. Valproate-ethosuximide combination therapy for refractory absence seizures. Archives of neurology. 1983 Dec;40(13):797-802. PubMed PMID: 6416232.

[16] Ferrie CD, Robinson RO, Knott C, Panayiotopoulos CP. Lamotrigine as an add-on drug in typical absence seizures. Acta neurologica Scandinavica. 1995 Mar;91(3):200-202. PubMed PMID: 7793236.

[17] Berkovic SF, Andermann F, Guberman A, Hipola D, Bladin PF. Valproate prevents the recurrence of absence status. Neurology. 1989 Oct;39(10):1294-1297. PubMed PMID: 2507956.

[18] Marson AG, Williamson PR, Clough H, Hutton JL, Chadwick DW, Epilepsy Monotherapy Trial G. Carbamazepine versus valproate monotherapy for epilepsy: a meta-analysis. Epilepsia. 2002 May:43(5):505-513. PubMed PMID: 12027911.

[19] Verrotti A, Grosso S, D'Egidio C, Parisi P, Spalice A, Pavone P, et al. Valproate in adolescents with photosensitive epilepsy with generalized tonic-clonic seizures only. European journal of paediatric neurology: EJPN: official journal of the European Paediatric Neurology Society. 2014 Jan;18(1):13-18. PubMed PMID: 23891468.

[20] Guerrini R. Valproate as a mainstay of therapy for pediatric epilepsy. Paediatr Drugs. 2006;8:113-129.

[21] Loscher W, Honack D. Valproate and its major metabolite E-2-envalproate induce different eff ects on behaviour and brain monoamine metabolism in rats. Eur I Pharmacol. 1996;299:61-67.

[22] Barry JJ. The Recognition and Management of Mood Disorders as a Comorbidity of Epilepsy. Epilepsia. 2003;44(S4):30-40.

[23] Klapper J. Divalproex sodium in migraine prophylaxis: A dose controlled study. Cephalalgia. 1997; 17:103-108.

[24] Freitag FG, Collins SD, Carlson HA, et al. A randomized trial of divalproex sodium extended-release tablets in migraine prophylaxis. Neurology. 2002:58:16521659.

[25] Yılmaz U, Yılmaz TS, Akıncı G, Korkmaz HA, Tekgül H. The effect of antiepileptic drugs on thyroid function in children. Seizure. 2014:23:29-35.

[26] Pylvanen V, Pakarinen A, Knip M, Isojarvi J. Characterization of insulin secretion in Valproate-treated patients with epilepsy. Epilepsia. 2006;47:1460-1464.

[27] Morrell MJ, Hayes FJ, Sluss PM, et al. Hyperandrogenism, ovulatory dysfunction and polycystic ovary syndrome with valproate versus lamotrigine. Ann Neurol 2008;64:200-211.

[28] Knorr M, Schaper J, Harjes M, et al. Fanconi syndrome caused by antiepileptic therapy with valproic acid. Epilepsia. 2004:45:868-871.

[29] Yacoob Y, Bhigjee AL, Moodley P, Parboosing R. Valproate and highly active antiretroviral therapy in HIV positive patients who develop new onset seizures. Seizure. 2011:20:80-82.

[30] Hsieh CY, Lai EC, Yang YH, Lin SJ. Comparative stroke risk of antiepileptic drugs in patients with epilepsy. Epilepsia. 2013;54:172-180.

[31] Perucca E. Clinically relevant drug interactions with antiepileptic drugs. Br J Clin Pharmacol. 2006 Mar:61:246-255.

[32] Acharya S, Bussel JB. Hematologic toxicity of valproate. J Pediatr Hematol Oncol. 2000;22:62-65.

[33] Murthy J. Tuberculous meningitis: The challenges. Neurol India. 2010;58:716722.

[34] Pohlmann-Eden B, Peters CN, Wennberg R, Dempfle CE. Valproate induces reversible factor XIII defi ciency with risk of perioperative bleeding. Acta Neurol Scand. 2003;108:142-145.

[35] Anderson GD, Lin YX, Berge C, Ojemann GA. Absence of bleeding complications in patients undergoing cortical surgery while receiving valproate treatment. J Neurosurg. 1997;87(2):252-256. 